\title{
Insulin resistance in Nigerians with essential hypertension
}

\author{
*Akande TO, Adeleye JO, Kadiri S \\ Department of Medicine, College of Medicine, University of Ibadan, Ibadan, Nigeria
}

\begin{abstract}
Background: Reports on the association between hypertension and insulin resistance have been inconsistent even though most studies show a definite association. It is also not certain if the association between insulin resistance and hypertension applies to all populations.

Objective: To determine the prevalence of insulin resistance in hypertensive Nigerians and to examine the association of insulin resistance with hypertension and some anthropometric indices.

Methods: Thirty five adults with essential hypertension and thirty five normotensives were studied. Anthropometric parameters, blood pressure, fasting glucose and insulin were measured. Homeostasis model assessment (HOMA) was used to determine insulin resistance (IR).

Results: The hypertensive subjects had significantly higher fasting insulin and HOMA-IR compared with normotensives ( $p=0.02$ and 0.04 ) respectively. There were significant correlations between HOMA-IR, BMI, waist and hip circumference in subjects with hypertension. At multiple linear regression, hypertension and body mass index were found to be the only significant predictors of insulin resistance.

Conclusion: The hypertensives we studied had a higher occurrence of insulin resistance compared to the normotensives. This makes it necessary for persons with hypertensive to have regular screening for diabetes and other categories of glucose intolerance as the increased insulin increases their risk of developing type 2 diabetes mellitus.

Keywords: Hypertension, Insulin resistance, Homeostasis model assessment

African Health Sciences 2013; 13(3): 655 - 660 http://dx.doi.org/10.4314/ahs.v13i3.19
\end{abstract}

\section{Introduction}

Hypertension is an important medical and public health problem in both developed and developing countries. It affects $25 \%$ of the adult population worldwide and its prevalence is predicted to increase by $60 \%$ by 2025 , when a total of 1.56 billion people may be affected ${ }^{1}$. Essential hypertension accounts for as many as $95 \%$ of cases of hypertension ${ }^{2}$. Hypertension and type 2 diabetes mellitus (DM) are interrelated metabolic disorders that strongly predispose an individual to macrovascular and microvascular complications. In recent years insulin resistance has been shown to play a central role in the development of hypertension, diabetes, obesity and dyslipidaemia.

Insulin resistance, a pathological situation characterised by reduced tissue sensitivity to insulin and marked compensatory hyperinsulinemia has continued to generate interest. It has been implicated in the pathogenesis of type 2 diabetes mellitus and

\begin{tabular}{l} 
*Corresponding author: \\
Dr Akande Temilola O \\
Department of Medicine, College of Medicine \\
University of Ibadan, Ibadan \\
Nigeria \\
Telephone: +2348035175482 \\
E-mail: fumkande@yahoo.com \\
\hline
\end{tabular}

African Health Sciences Vol 13 Issue 3 September 2013 essential hypertension and is closely associated with dyslipidaemia, coronary artery disease, obesity and a cluster of metabolic and cardiovascular abnormalities that define the metabolic syndrome ${ }^{3 \text {, }}$ 4.

The progression from normal glucose tolerance to type 2 diabetes mellitus is characterised by dual defects that include insulin resistance and an insulin secretory defect caused by beta cell dysfunction ${ }^{5}$. Insulin resistance is said to precede the development of impaired glucose tolerance (IGT) and type $2 \mathrm{DM}$ by decades ${ }^{3}$. Identifying persons with insulin resistance who are therefore at risk for developing type $2 \mathrm{DM}$ would afford an opportunity for intervention and possible delay or prevention of diabetes.

Insulin resistance can be estimated using several techniques. The euglycaemic hyperinsulinemic clamp technique is the gold standard method for evaluation ${ }^{6}$. However this method is complex and expensive. HOMA-IR is a simple and reliable surrogate measure of insulin resistance, ${ }^{7}$. HOMA model is derived from a mathematical assessment of the interaction between beta cell function and insulin resistance in an idealized model that is then used to compute steady state insulin and glucose 
concentrations ${ }^{9,10}$. An advantage of the HOMA method is that only a single venepuncture is required so it is simple and easy to use.

Reports on the association between hypertension and insulin resistance have been inconsistent and it is not certain if the association between insulin resistance and hypertension applies to all populations. There is also paucity of data on insulin resistance among Nigerians with hypertension.

This study aims to determine the prevalence of insulin resistance in a group of Nigerians with essential hypertension using the HOMA method and to examine the relationship between insulin resistance, hypertension and some anthropometric indices.

\section{Methods}

The study population comprised of 70 adults. Thirty five consecutive persons with essential hypertension seen at the General Outpatient Clinics of a University Teaching Hospital formed the study subjects while 35 persons who did not have a history of hypertension or diabetes and whose measured blood pressures were normal were recruited as controls. Controls were recruited consecutively from hospital staff and relatives of our patients.

Hypertension was diagnosed if the systolic blood pressure was equal to or greater than $140 \mathrm{mmHg}$ and or if the diastolic blood pressure was equal to or greater than $90 \mathrm{mmHg}$ recorded on at least two occasions or if the person was on antihypertensive medications. ${ }^{11}$ Subjects with secondary hypertension and persons already diagnosed to have diabetes were excluded from the study. Other exclusion criteria included pregnant women and persons with acute intercurrent illness or persons receiving medications known to affect glucose metabolism. Participants were told to be on their usual diet for the three days prior to the oral glucose tolerance test. Ethical clearance was obtained from the local ethics committee and written informed consent was obtained from all subjects before participation.

All persons studied had information on age, gender, tribe and marital status recorded in addition to weight, height, waist and hip circumference, and blood pressure. Body mass index (BMI) was calculated as the weight in kilograms divided by the height in meters squared. Obesity was defined as a BMI $>30 \mathrm{~kg} / \mathrm{m}^{2}$ while truncal obesity was defined as a waist circumference $>94 \mathrm{~cm}$ in males and $>80 \mathrm{~cm}$ in females. Waist and hip circumference $(\mathrm{cm})$ were measured with a flexible inelastic tape and the waist to hip ratio was calculated. Blood pressure was measured using a sphygnomanometer in the sitting position after a five minutes rest.

\section{Metabolic studies}

Following an overnight fast of 10-12 hours, four mls of venous blood was drawn from each participant and divided into equal aliquots for analysis of the fasting plasma glucose and fasting insulin. The blood collected for glucose analysis was promptly centrifuged and analyzed by a glucose oxidase method with kits from Dialab Diagnostics, Austria while the blood collected for insulin estimation was centrifuged and stored at $-20^{\circ} \mathrm{C}$ until analyzed. Serum insulin levels were determined by Enzyme Linked Immunosorbent Assay method using instruments and kits from Diagnostic Automation Incorporated, Canada. The kit has no cross reactivity with intact human proinsulins. The intra-assay and inter-assay coefficients of variation were 6.29 and 7.67 respectively.

Insulin resistance was derived using the homeostasis model assessment method and was calculated using the formula: HOMA-IR = Fasting insulin value $(\mathrm{mU} / \mathrm{L}) \mathrm{X}$ Fasting plasma glucose $(\mathrm{mmol} / \mathrm{L}) / 22.5^{9}$. Persons with HOMA-IR $>3.8$ were considered to have insulin resistance ${ }^{12}$.

Data were analyzed with SPSS version 16. Continuous variables were presented as mean (standard deviation) and categorical values as no (\%). Comparison between means was done using the $t$ test for two groups and analysis of variance for more than two groups. Associations between two categorical variables were tested using the Chi square test. Level of significance was p value less than 0.05 .

\section{Results}

A total of 35 hypertensives and 35 normotensives were studied. The characteristics of the subjects and controls are summarised in table 1 . The mean age of the subjects with hypertension was $55.8 \pm 10.3$ years while the mean age of the controls was $50.5 \pm$ 9.1 years $(p=0.026)$. The age range of hypertensives and controls was $37-80$ years and 35 to 75 years respectively. 
Table 1: Clinical and metabolic characteristics of hypertensive and control subjects

\begin{tabular}{|c|c|c|c|}
\hline Parameter & $\begin{array}{l}\text { Hypertensives } \\
(\mathrm{n}=35) \text { Mean (SD) }\end{array}$ & $\begin{array}{l}\text { Controls } \\
(n=35) \text { Mean (SD) }\end{array}$ & $\mathbf{P}$ value \\
\hline Systolic blood pressure (mmHg) & $142.5 \pm 16.2$ & $115.8 \pm 11.5$ & $0.001 *$ \\
\hline Diastolic blood pressure $(\mathrm{mmHg})$ & $94.5 \pm 10.7$ & $76.4 \pm 7.3$ & $<0.001^{*}$ \\
\hline Weight $(\mathrm{kg})$ & $73.6 \pm 14.6$ & $74.3 \pm 15.8$ & 0.85 \\
\hline BMI $(\mathrm{kg} / \mathrm{m} 2)$ & $27.4 \pm 5.8$ & $26.8 \pm 5.3$ & 0.69 \\
\hline Waist Circumference $(\mathrm{cm})$ & $93.6 \pm 13.7$ & $90.0 \pm 11.8$ & 0.26 \\
\hline Waist: Hip ratio & $0.91 \pm 0.1$ & $0.88 \pm 0.1$ & 0.17 \\
\hline FPG (mg/dl) & $93.1 \pm 19.9$ & $89.9 \pm 13.3$ & 0.43 \\
\hline FPI (mU/ml) & $15.5 \pm 5.3$ & $12.9 \pm 3.4$ & $0.02 *$ \\
\hline HOMA-IR & $3.6 \pm 1.9$ & $2.9 \pm 0.9$ & $0.04 *$ \\
\hline
\end{tabular}

${ }^{*} \mathrm{p}<0.05$

$\mathrm{BMI}=$ body mass index, $\mathrm{FPG}=$ fasting plasma glucose, $\mathrm{FPI}=$ fasting plasma insulin, $\mathrm{HOMA}-\mathrm{IR}=$ Homeostasis model assessment of insulin resistance

The systolic and diastolic blood pressures in the hypertensives ranged from $100-182 \mathrm{mmHg}$ and 72 to $115 \mathrm{mmHg}$ respectively, while in the normotensives it ranged from 90-132mmHgand 58$85 \mathrm{mmHg}$ respectively. The mean systolic and diastolic blood pressures in the hypertensive group was $142.5 \pm 16.2 \mathrm{mmHg}$ and $94.5 \pm 10.7 \mathrm{mmHg}$ respectively $(\mathrm{p}=0.001)$ while it was $115.8 \pm 11.5$ $\mathrm{mmHg}$ and $76.4 \pm 7.3 \mathrm{mmHg}$ in the control group $(\mathrm{p}<0.001)$.

Eleven $(31.4 \%)$ of the hypertensives and $8(22.9 \%)$ of the normotensives were obese $(\mathrm{p}=0.42)$. Truncal obesity was present in $71.4 \%$ of hypertensive subjects and $68.6 \%$ of the controls though these did not attain statistical significance. There was no statistically significant difference in anthropometric indices between hypertensives and controls even when analysed according to gender.

Fasting plasma glucose did not differ significantly between the 2 groups though fasting insulin concentration and HOMA-IR were significantly higher in the hypertensive group compared with the control group ( $\mathrm{p}=0.02$ and 0.04 respectively). $31.4 \%$ of hypertensives and $8.6 \%$ of normotensives had insulin resistance $(\mathrm{p}=0.02)$.

There were significant correlations between HOMAIR and BMI $(r=0.603, p<0.001)$, waist circumference $(\mathrm{r}=0.477, \mathrm{p}<0.004)$ and hip circumference $(\mathrm{r}=0.519$, $\mathrm{p}<0.001)$ in subjects with hypertension. HOMA-IR also correlated significantly with waist circumference $(\mathrm{r}=0.399, \mathrm{p}=0.02)$ and hip circumference $(\mathrm{r}=0.383$, $\mathrm{p}=0.02)$ in controls.

At multiple linear regression, after adjustment for age, BMI, waist circumference and blood pressure, hypertension and BMI were found to be the only significant predictors of insulin resistance (regression coefficient $0.736,95 \%$ confidence interval 0.038 $1.434, \mathrm{p}=0.04$ and regression coefficient 0.115 , confidence interval $0.052-0.177, \mathrm{p}=0.001$ respectively) (table 2).

Table 2: Linear regression analysis showing factors associated with the presence of insulin resistance

\begin{tabular}{llll}
\hline & $\begin{array}{l}\text { Regression } \\
\text { coefficient } \\
\text { (Beta) }\end{array}$ & $\begin{array}{l}\mathbf{9 5 \%} \text { CI for } \\
\text { (Beta) }\end{array}$ & P value \\
\hline Age & 0.007 & $-0.034-0.047$ & 0.74 \\
Hypertension & 0.736 & $0.038-1.434$ & $0.04^{*}$ \\
BMI & 0.115 & $0.052-0.177$ & $0.001^{*}$ \\
Waist circumference & 0.023 & $-0.015-0.062$ & 0.23 \\
\hline
\end{tabular}

$*_{\mathrm{p}}<0.05$

$\mathrm{CI}=$ confidence interval, $\mathrm{BMI}=$ body mass index 
A higher percentage of persons using thiazide diuretics compared with those not using thiazide diuretics had insulin resistance $(\mathrm{p}=0.032)$. For other antihypertensives (calcium channel blockers, angiotensin-converting enzyme inhibitors and centrally acting drugs) being used, there was no significant difference in insulin resistance between users and non-users (table 3).

Table 3: Relationship between antihypertensive use and presence of insulin resistance

\begin{tabular}{llllll}
\hline Drug type & $\begin{array}{l}\text { Insulin } \\
\text { resistant } \\
\mathbf{n}(\%)\end{array}$ & $\begin{array}{l}\text { Not insulin } \\
\text { resistant } \\
\mathbf{n}(\%)\end{array}$ & Total & $\mathbf{C h i}^{2}$ & P value \\
\hline Thiazides & $7(13.7)$ & $44(86.3)$ & 51 & & \\
No & $7(36.8)$ & $12(63.2)$ & 19 & 4.62 & $0.032^{*}$ \\
Yes & & & & & \\
Calcium channel blockers & $8(16.7)$ & $40(83.3)$ & 48 & & \\
No & $6(27.3)$ & $16(72.7)$ & 22 & 1.06 & 0.303 \\
Yes & $12(19)$ & $51(81)$ & 63 & & \\
ACEIs & $2(25)$ & $6(75)$ & 8 & 0.159 & 0.690 \\
No & $12(18.8)$ & $52(81.2)$ & 64 & & \\
Yes & $2(33.3)$ & $4(66.7)$ & 6 & 0.729 & 0.393 \\
Centrally acting drugs & & & & & \\
No & & & & & \\
Yes & & & & & \\
\hline
\end{tabular}

$*_{\mathrm{p}}<0.05$

ACEIs - angiotensin-converting enzyme inhibitors

\section{Discussion}

In our study fasting insulin concentration and HOMA-IR were significantly higher in the hypertensives compared with the normotensives. Insulin resistance was present in a higher proportion of hypertensives $(31.4 \%$ of hypertensives compared to $8.6 \%$ of controls), indicating an increased risk of subsequent development of type $2 \mathrm{DM}$ in the hypertensives since insulin resistance is well recognised as an independent predictive factor for subsequent development of type 2 DM. Our findings are consistent with those from other African populations which have shown that persons with essential hypertension are more insulin resistant and hyperinsulinemic compared to normotensive persons (13-15). Similar findings have also been reported amongst Iranians with hypertension ${ }^{13}$.

Studies have shown prevalence of insulin resistance in hypertensives ranging between $27-50 \%{ }^{14,15}$. However different methods and different cut offs were used in assessing insulin resistance in the various studies. This may make comparisons between studies difficult. ${ }^{16,17}$.

Though our study showed that insulin resistance was commoner in hypertensives, we also found that 8.6\% of our normotensives had insulin resistance. This is similar to findings by Bonora et al where insulin resistance was found in $9.6 \%$ of the general population ${ }^{18}$. Studies have shown that increasing waist circumference, waist hip ratio and BMI are associated with increasing insulin resistance, hyperinsulinemia and glucose intolerance ${ }^{19}$. It is noteworthy that the mean BMI amongst control subjects was in the overweight range. In addition, the control subjects did not differ significantly from the hypertensive subjects as regards the anthropometric indices. Apart from environmental factors such as overweight and obesity, researchers have shown that racial and genetic factors have a role to play in the development of insulin resistance. It is probable that some of our controls may have a genetic predisposition to insulin resistance or had unrecognized or undiagnosed first degree relative with hypertension or type 2 diabetes making them to be at an increased risk for insulin resistance 20,21 .

In a study in Ibadan, where this study was done, prevalence of obesity was found to be $16.7 \%$ among relatively healthy persons ${ }^{22}$. In our study obesity was present in $22.9 \%$ of the control population. The higher prevalence we obtained for obesity in our normotensives may be partly due to the fact that some of them were first degree relatives of hypertensives putting them at risk of the metabolic syndrome of which obesity is a component and may

African Health Sciences Vol 13 Issue 3 September 2013 
also signify a rise in incidence of obesity as is occurring in some developed nations.

Increased abdominal fat distribution has been associated with increased incidence of essential hypertension, type 2 diabetes mellitus and insulin resistance ${ }^{19}$. In this study, the greater frequency of occurrence of insulin resistance along with higher BMI, waist circumference and waist hip ratios in hypertensive when compared to controls would support the concept of the metabolic syndrome in which there is a clustering of various cardiovascular risk factors and consequently a higher cardiovascular risk.

Studies have shown that insulin resistance is associated with hypertension and a pathogenetic role in the development of hypertension has been suggested. There is however still debate regarding the role and mechanism of insulin resistance in hypertension. Several mechanisms have been adduced. One is that insulin resistance contributes to the development of essential hypertension as it leads to hyperinsulinemia which can increase arterial pressure by causing renal sodium retention and increased sympathetic activity ${ }^{23}$. Another explanation is that insulin resistance and the resultant hyperinsulinemia can lead to renin angiotensin system activation and intracellular calcium accumulation in vascular smooth muscle thus leading to elevated blood pressures ${ }^{24}$. It is however not certain whether insulin resistance precedes or is an outcome of hypertension. This remains to be demonstrated by more studies aimed at determining the mechanistic link between insulin resistance and hypertension which this study did not set out to determine.

Among our study population, insulin resistance was significantly more in persons using thiazide diuretics. This has implication on choice of antihypertensive medications and it would be desirable to avoid or use thiazides in low doses in hypertensives that have additional risk factors for diabetes (obesity, first degree relative with diabetes or other components of the metabolic syndrome). Our findings also suggest a need for larger studies to corroborate our findings and to determine the mechanistic link between hypertension, insulin resistance and diabetes in the general population. Even though the precise mechanisms of insulin resistance in hypertensives may not be clear, studies have demonstrated the benefit of early interventions to improve insulin sensitivity in these persons ${ }^{25}$. Strategies to reduce insulin resistance such as physical exercise, weight loss and a healthy diet should be included in the treatment of hypertensive persons in order to ameliorate insulin resistance and prevent the development of type 2 diabetes. A major limitation of our study was the small sample size studied which was mainly due to limited funds.

\section{Conclusion}

We have shown that insulin resistance occurred more frequently in the hypertensives compared to the normotensives studied, and that hypertension and increased BMI were the only significant predictors of insulin resistance. We also showed a positive correlation between insulin resistance, BMI and waist circumference. Our findings may also have potential implications with regards to preventive strategies for amelioration of insulin resistance and prevention of type $2 \mathrm{DM}$ amongst persons with systemic hypertension.

\section{Acknowledgment}

The authors would like to acknowledge the contributions of the following people: Dr J. O Yaria for patiently entering the data and Dr B. O Adedokun and Dr A. M Adebayo for assistance with data analysis.

\section{References}

1. Kearney PM, Whelton M, Reynolds K, Muntner P, Whelton PK and He J. Global burden of hypertension: analysis of worldwide data. Lancet. 2005; 365: 217-23.

2. Wofford MR, King DS, Wyatt SB and Jones DW. Secondary Hypertension: Detection and Management for the Primary Care Provider. $J$ Clin Hypertens (Greenwich). 2000; 2: 124-31.

3. Rader DJ. Effect of insulin resistance, dyslipidemia, and intra-abdominal adiposity on the development of cardiovascular disease and diabetes mellitus. The American journal of medicine. 2007; 120: S12-8.

4. Reaven GM. Insulin resistance and compensatory hyperinsulinemia: role in hypertension, dyslipidemia, and coronary heart disease. American heart journal. 1991; 121: 1283-8.

5. Ramlo-Halsted BA and Edelman SV. The natural history of type 2 diabetes. Implications for clinical practice. Prim Care. 1999; 26: 771-89.

6. Ferrannini E and Mari A. How to measure insulin sensitivity. Journal of hypertension. 1998; 16: 895-906. 
7. Ikeda Y, Suehiro T, Nakamura T, Kumon Y and Hashimoto K. Clinical significance of the insulin resistance index as assessed by homeostasis model assessment. Endocrine journal. 2001; 48: 816.

8. Bonora E, Targher G, Alberiche M, et al. Homeostasis model assessment closely mirrors the glucose clamp technique in the assessment of insulin sensitivity: studies in subjects with various degrees of glucose tolerance and insulin sensitivity. Diabetes care. 2000; 23: 57-63.

9. Matthews DR, Hosker JP, Rudenski AS, Naylor BA, Treacher DF and Turner RC. Homeostasis model assessment: insulin resistance and betacell function from fasting plasma glucose and insulin concentrations in man. Diabetologia. 1985; 28: 412-9.

10. Wallace TM, Levy JC and Matthews DR. Use and abuse of HOMA modeling. Diabetes care. 2004; 27: 1487-95.

11. Chobanian AV, Bakris GL, Black HR, et al. Seventh report of the Joint National Committee on Prevention, Detection, Evaluation, and Treatment of High Blood Pressure. Hypertension. 2003; 42: 1206-52.

12. Garcia-Puig J, Ruilope LM, Luque M, Fernandez J, Ortega R and Dal-Re R. Glucose metabolism in patients with essential hypertension. The American journal of medicine. 2006; 119: 318-26.

13. Esteghamati A, Khalilzadeh O, Abbasi M, Nakhjavani M, Novin L and Esteghamati AR. HOMA-estimated insulin resistance is associated with hypertension in Iranian diabetic and nondiabetic subjects. Clin Exp Hypertens. 2008; 30: 297-307.

14. Lind L, Berne C and Lithell H. Prevalence of insulin resistance in essential hypertension. Journal of hypertension. 1995; 13: 1457-62.

15. Lima NK, Abbasi F, Lamendola $C$ and Reaven GM. Prevalence of insulin resistance and related risk factors for cardiovascular disease in patients with essential hypertension. Am J Hypertens. 2009; 22: 106-11.
16. Saad MF, Lillioja S, Nyomba BL, et al. Racial differences in the relation between blood pressure and insulin resistance. $N$ Engl J Med. 1991; 324: 733-9.

17. Saad MF, Rewers M, Selby J, et al. Insulin resistance and hypertension: the Insulin Resistance Atherosclerosis study. Hypertension. 2004; 43: 1324-31.

18. Bonora E, Kiechl S, Willeit J, et al. Prevalence of insulin resistance in metabolic disorders: the Bruneck Study. Diabetes. 1998; 47: 1643-9.

19. Ferrannini E, Haffner SM, Mitchell BD and Stern MP. Hyperinsulinaemia: the key feature of a cardiovascular and metabolic syndrome. Diabetologia. 1991; 34: 416-22.

20. Facchini F, Chen YD, Clinkingbeard C, Jeppesen $\mathrm{J}$ and Reaven GM. Insulin resistance, hyperinsulinemia, and dyslipidemia in nonobese individuals with a family history of hypertension. Am J Hypertens. 1992; 5: 694-9.

21. Amoah AG, Owusu SK, Ayittey OM, Schuster DP and Osei K. Minimal model analyses of beta cell secretion, insulin sensitivity and glucose effectiveness in glucose tolerant, non-diabetic first-degree relatives of Ghanaian patients with type 2 diabetes and healthy control subjects. Ethnicity \& disease. 2001; 11: 201-10.

22. Balogun MO and Owoaje ET. Work conditions and health problems of female traders in Ibadan, Nigeria. African journal of medicine and medical sciences. 2007; 36: 57-63.

23. Reaven GM and Hoffman BB. A role for insulin in the aetiology and course of hypertension? Lancet. 1987; 2: 435-7.

24. Ferrannini E, Santoro D and Manicardi V. The association of essential hypertension and diabetes. Comprehensive therapy. 1989; 15: 51-8.

25. Osei K. Insulin resistance and systemic hypertension. The American journal of cardiology. 1999; 84: 33J-6J. 